\title{
ON INVARIANT DOMAINS OF HOLOMORPHY
}

\author{
A. G. SERGEEV \\ Steklov Mathematical Institute, Vavilova 42, 117966, GSP-1, Moscow, Russia \\ E-mail: armen@sergeev.mian.su
}

Let $D$ be a domain in $\mathbb{C}^{n}$ and $\mathcal{F}=\mathcal{F}(D)$ be a subclass in the class $\mathcal{O}=$ $\mathcal{O}(D)$ of functions holomorphic in $D$. Recall that $D$ is called an $\mathcal{F}$-domain of holomorphy iff there exists a function $f \in \mathcal{F}(D)$ which cannot be holomorphically extended across the boundary of $D$. If, for example, we take for $\mathcal{F}(D)$ the class $\mathcal{O}_{\text {temp }}(D)$ of temperate holomorphic functions in $D$ (i.e. holomorphic functions in $D$ growing less than a power of distance to the boundary of $D$ ) then the notion

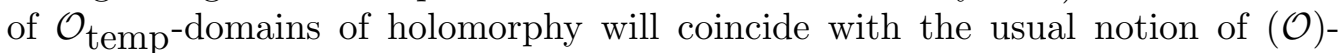
domains of holomorphy according to Pflug [10]. On the other hand, considering as $\mathcal{F}(D)$ the class $H^{\infty}(D)$ of bounded holomorphic functions we obtain the notion of $H^{\infty}$-domains of holomorphy which is quite different from the notion of $(\mathcal{O})$ domains of holomorphy (cf. Sibony [13]). In this paper we are interested in the case when $D$ is invariant under the action of a compact Lie group $K$ and $\mathcal{F}(D)$ coincides with the class $\mathcal{O}^{K}(D)$ of $K$-invariant holomorphic functions in $D$. From first examples of $\mathcal{O}^{K}$-domains of holomorphy it becomes clear that this notion differs much from the usual notion of domains of holomorphy. Consider, e.g., the ring $D=\{1<|z|<2\}$ in $\mathbb{C}^{1}$ with the action of the circle group $S^{1}$ given by rotations. Then the only $S^{1}$-invariant holomorphic functions in $D$ are constants so they extend holomorphically across the boundary of $D$ to all of $\mathbb{C}^{1}$ (note that $D$ is a domain of holomorphy in this example). Later on we shall give several (less trivial) examples of that sort. This article based on recent results by Peter Heinzner, Xiangyu Zhou and the author (cf. [4], [5], [12], [16]) contains some general assertions about $\mathcal{O}^{K}$-domains of holomorphy and their holomorphic hulls

1991 Mathematics Subject Classification: 32D05, 32H20.

Key words and phrases: complexification, orbit convexity, orbit connectedness, matrix Reinhardt domains, extended future tube.

Research partially supported by a grant NFT000 from the International Science Foundation and grant 93-011-140 from the Russian Foundation of Fundamental Research.

The paper is in final form and no version of it will be published elsewhere. 
with respect to $K$-invariant holomorphic functions (Section I). In Sec. II we apply these general assertions to some particular $K$-invariant domains such as matrix Reinhardt domains and the extended matrix disc. In order to avoid technicalities and present the ideas rather than results in their full generality we restrict here to the case of a compact connected Lie group $K$ acting linearly on $\mathbb{C}^{n}$. The general case of a compact (maybe, not connected) Lie group acting holomorphically on a Stein space is considered in Heinzner [4].

\section{Complexification of invariant domains of holomorphy}

1. Complexification of a compact Lie group. We assume throughout this paper that $K$ is a compact connected real Lie group. The complexification of $K$ (cf. Hochschild [6]) is a complex Lie group $K^{\mathbb{C}}$ with a (continuous) homomorphism $i$ : $K \rightarrow K^{\mathbb{C}}$ such that for any (continuous) homomorphism $\varphi: K \rightarrow G$ to a complex Lie group $G$ there exists a unique holomorphic homomorphism $\psi: K^{\mathbb{C}} \rightarrow G$ closing the commutative diagram

$$
\begin{array}{ccc}
K & \stackrel{i}{\rightarrow} & K^{\mathbb{C}} \\
\varphi \searrow & & \swarrow \psi \\
& G &
\end{array}
$$

We list now some basic properties of group complexifications (cf. Hochschild [6]).

(i) The complexification $K^{\mathbb{C}}$ is uniquely defined up to biholomorphic homomorphisms.

(ii) The Lie algebra $\mathfrak{k}^{\mathbb{C}}$ of $K^{\mathbb{C}}$ is the complexification of the Lie algebra $\mathfrak{k}$ of $K$, i.e. $\mathfrak{k}^{\mathbb{C}}=\mathfrak{k}+i \mathfrak{k}$.

(iii) $K^{\mathbb{C}}$ is Stein and $i(K)$ is a totally real submanifold of $K^{\mathbb{C}}$ with $\operatorname{dim}_{\mathbb{R}} i(K)$ $=\operatorname{dim}_{\mathbb{C}} K^{\mathbb{C}}$

ExAmples. $1 . \quad K=S^{1} \Rightarrow K^{\mathbb{C}}=\mathbb{C}^{*}=\mathbb{C} \backslash 0 \quad$ (multiplicative group of complex numbers),

2. $K=\mathrm{SU}(n) \Rightarrow K^{\mathbb{C}}=\mathrm{SL}(n, \mathbb{C})$, $K=\mathrm{U}(n) \Rightarrow K^{\mathbb{C}}=\mathrm{GL}(n, \mathbb{C})$.

2. Complexification of invariant domains. Let the group $K$ act linearly on $\mathbb{C}^{n}$, i.e. the action of $K$ on $\mathbb{C}^{n}$ is given by a representation $\rho: K \rightarrow \operatorname{GL}\left(\mathbb{C}^{n}\right)$. By the definition of complexification this representation generates a holomorphic representation $\rho^{\mathbb{C}}: K^{\mathbb{C}} \rightarrow \mathrm{GL}\left(\mathbb{C}^{n}\right)$, i.e. a holomorphic linear action of $K^{\mathbb{C}}$ on $\mathbb{C}^{n}$.

Definition. Let $D$ be a $K$-invariant domain in $\mathbb{C}^{n}$. We call its complexification the domain

i.e. the image of $D$ under $K^{\mathbb{C}}$-action.

$$
D_{\mathbb{C}}=K^{\mathbb{C}} \cdot D,
$$

Note that $D_{\mathbb{C}}$ is really a domain (i.e. an open connected set) if $D$ is a domain. The above definition agrees with the general definition of the complexification 
of a Stein space given in Heinzner [4]. We are going to show (cf. Theorems 1, 2) that under some natural conditions on the $K$-action on $D$ its complexification $D_{\mathbb{C}}$ coincides with the holomorphic hull of $D$ with respect to invariant holomorphic functions.

ExAmple. Reinhardt domains in $\mathbb{C}^{n}$ are the domains invariant under the action of the torus group $\left(S^{1}\right)^{n}$, i.e. domains $D \subset \mathbb{C}^{n}$ satisfying the condition

$$
\left(z_{1}, \ldots, z_{n}\right) \in D \Rightarrow\left(e^{i \theta_{1}} z_{1}, \ldots, e^{i \theta_{n}} z_{n}\right) \in D
$$

for all $\left(z_{1}, \ldots, z_{n}\right) \in D$ and all real $\theta_{1}, \ldots, \theta_{n}$. The complexification of a Reinhardt domain $D$ is the domain

$$
D_{\mathbb{C}}=\left(\mathbb{C}^{*}\right)^{n} \cdot D=\left\{\left(\lambda_{1} z_{1}, \ldots, \lambda_{n} z_{n}\right):\left(z_{1}, \ldots, z_{n}\right) \in D ; \lambda_{j} \in \mathbb{C}^{*}, 1 \leq j \leq n\right\}
$$

which coincides with the direct product $\left(\mathbb{C}^{*}\right)^{r} \times \mathbb{C}^{n-r}$ for some $r, 0 \leq r \leq n$. Other examples of complexifications will be given in Sec. 2.

3. Orbit convexity. Main results on complexifications $D_{\mathbb{C}}$ of $K$-invariant domains $D$ will be proved under a condition that orbits of $K^{\mathbb{C}}$ in $D_{\mathbb{C}}$ are "wellbehaved". Roughly speaking, the "good behaviour" of $K^{\mathbb{C}}$-orbits means the following. First, since $D$ is invariant under $K$ we need to look only at the orbits of $K^{\mathbb{C}}$ going through points of $D$ in the "orthogonal directions", i.e. orbits of $K^{\mathbb{C}}$ tangent to the Lie subalgebra $i \mathfrak{k}$ in $\mathfrak{k}^{\mathbb{C}}$. Their "good behaviour" means that different orbits should not meet outside $D$. More precisely, we have the following definition.

Definition (Heinzner [4]). Let $D$ be a $K$-invariant domain in $\mathbb{C}^{n}$. It is called orbit convex if for any $z \in D$ and any $v \in i \mathfrak{k}$ the inclusion $\exp v \cdot z \in D$ implies that

$$
\exp (t v) \cdot z \in D \quad \text { for } 0 \leq t \leq 1 .
$$

Here $\exp : \mathfrak{k}^{\mathbb{C}} \rightarrow K^{\mathbb{C}}$ is the exponential mapping.

ExAmple. A Reinhardt domain in $\left(\mathbb{C}^{*}\right)^{n}$ is orbit convex if and only if it is $\log$ (logarithmically)-convex.

Further examples of orbit convex domains will be given in Sec. 2, now we want to exploit the orbit convexity for the investigation of complexifications $D_{\mathbb{C}}$.

4. Holomorphic extension. We have the following extension theorem for $K$ invariant functions.

Theorem 1. Let $D$ be a $K$-invariant orbit convex domain in $\mathbb{C}^{n}$. Then any $K$-invariant holomorphic function $f$ on $D$ can be extended to a $K^{\mathbb{C}}$-invariant holomorphic function $\hat{f}$ on $D_{\mathbb{C}}$. Hence, $D_{\mathbb{C}}$ is a natural holomorphic extension of $D$ with respect to $K$-invariant holomorphic functions.

The theorem in this form was proved in Heinzner-Sergeev [5] and in a more general situation - in Heinzner [4]. For the extension of $f$ to $D_{\mathbb{C}}$ one needs to use 
the orbit convexity condition and identity principle to a holomorphic function on the totally real subset $i(K)$ in $K^{\mathbb{C}}$.

Remarks. 1. In Theorem 1 one can substitute $K$-invariant holomorphic functions $f$ in $D$ by $K$-equivariant holomorphic mappings $f: D \rightarrow Y$ to some holomorphic $K^{\mathbb{C}}$-manifold $Y$. The assertion is still true, i.e. such a mapping extends to a $K^{\mathbb{C}}$-equivariant holomorphic mapping $\hat{f}: D_{\mathbb{C}} \rightarrow Y$. Here, a mapping $f: D \rightarrow Y$ is called $K$-equivariant iff $f(k \cdot z)=k \cdot f(z)$ for any $z \in D, k \in K$; the $K^{\mathbb{C}}$-equivariance of $\hat{f}: D_{\mathbb{C}} \rightarrow Y$ is defined analogously.

2. There is a partial converse to Theorem 1 proved in Heinzner [4]. Suppose that $D$ is a $K$-invariant domain of holomorphy in $\mathbb{C}^{n}$ and $\Omega$ is a $K^{\mathbb{C}}$-invariant domain in $\mathbb{C}^{n}$ such that any $K$-equivariant holomorphic map $f: D \rightarrow V$ to a finite-dimensional representation space $V=\mathbb{C}^{k}$ extends to a holomorphic $K^{\mathbb{C}}$ equivariant map $\hat{f}: \Omega \rightarrow V$. Then $D$ is orbit convex.

5. Holomorphic convexity of $D_{\mathbb{C}}$. According to Theorem $1 K$-invariant holomorphic functions from an orbit convex domain $D$ extend to $D_{\mathbb{C}}$. The natural question is whether they could be extended further, or, to put it in another way, whether $D_{\mathbb{C}}$ is the holomorphic hull of $D$ with respect to $K$-invariant holomorphic functions. The answer is positive for orbit convex domains $D$.

TheOREM 2. Let $D$ be a $K$-invariant orbit convex domain of holomorphy in $\mathbb{C}^{n}$. Then $D_{\mathbb{C}}$ is also a domain of holomorphy which represents the holomorphic hull of $D$ with respect to $K$-invariant holomorphic functions.

This theorem is proved in Heinzner-Sergeev [5] (assuming thet $D_{\mathbb{C}}$ is saturated) and in Heinzner [4] in a more general setting. The proof is based on an invariant form of Cartan's theorem formulated next.

CARTAN's TheOREm. Let $D$ be a $K$-invariant domain of holomorphy in $\mathbb{C}^{n}$ and $A$ is a $K$-invariant analytic subset in $D$. Then any $K$-invariant analytic function $f$ on $A$ can be extended to a $K$-invariant holomorphic function $F$ on $D$ so that $F \mid A=f$.

For a compact Lie group $K$ this invariant version of Cartan's theorem follows immediately from the usual Cartan theorem. Indeed, using the last theorem we extend $f$ to a holomorphic function in $D$ and then integrate it over the group $K$. The integrated function $F$ is the one we are looking for.

Theorems 1 and 2 give a description of $\mathcal{O}^{K}$-holomorphic hulls of domains of holomorphy in $\mathbb{C}^{n}$ under the assumption that they are orbit convex. Thus we are motivated to study more carefully the orbit-convexity condition.

6. Orbit connectedness. First we note that the orbit-convexity condition can be slightly weakened without violating the assertions of Theorems 1 and 2. Namely, this condition can be substituted by a condition of orbit-connectedness which is formulated (in contrast with the orbit-convexity condition referring only to orbits tangent to $i \mathfrak{k})$ in terms of full $K^{\mathbb{C}}$-orbits of points in $D$. 
Definition (Heinzner [4]). Denote for $z \in \mathbb{C}^{n}$ by $b_{z}: K^{\mathbb{C}} \rightarrow \mathbb{C}^{n}$ the orbit map $b_{z}(h)=h \cdot z, h \in K^{\mathbb{C}}$. A $K$-invariant domain $D$ in $\mathbb{C}^{n}$ is called orbit connected iff the preimage

$$
b_{z}^{-1}(D)=\left\{h \in K^{\mathbb{C}}: h \cdot z \in D\right\}
$$

is connected in $K^{\mathbb{C}}$ for any $z \in \mathbb{C}^{n}$.

It follows from the polar decomposition of $K^{\mathbb{C}}$ that the orbit-convexity of $D$ implies its orbit-connectedness.

The same proof as in Theorem 1 applied to $K$-invariant orbit connected domains in $\mathbb{C}^{n}$ yields the assertion of this theorem for such domains. So from the partial converse to Theorem 1 (cf. Remark 2 after this theorem) we obtain that for $K$-invariant domains of holomorphy the orbit-connectedness implies orbitconvexity. Hence in this case both notions are equivalent and Theorem 2 is also true for $K$-invariant orbit connected domains of holomorphy.

Zhou has proved recently an extension of Theorem 2 for orbit connected domains which are not holomorphically convex.

Theorem 3 (Zhou [16]). Let $D$ be a $K$-invariant orbit connected domain in $\mathbb{C}^{n}$. Then its holomorphic hull $E(D)$ is schlicht and orbit connected $\Leftrightarrow E\left(D_{\mathbb{C}}\right)$ is schlicht. Moreover, in this case

$$
E\left(D_{\mathbb{C}}\right)=K^{\mathbb{C}} \cdot E(D) .
$$

In the next section we shall give further results on orbit-convexity and orbit-connectedness and consider non-trivial examples of domains satisfying these conditions.

\section{Orbit convex and orbit connected domains. Applications}

1. Orbit pseudoconvex domains. Let $D$ be a $K$-invariant domain in $\mathbb{C}^{n}$ given in the form

$$
D=\left\{z \in \mathbb{C}^{n}: \varphi(z)<0\right\}
$$

where $\varphi$ is a $K$-invariant real $C^{2}$-smooth function on $\mathbb{C}^{n}$.

Definition (Heinzner-Sergeev [5]). The function $\varphi$ is called orbit plurisubharmonic (with respect to $K^{\mathbb{C}}$-action on $\mathbb{C}^{n}$ ) if the Levi form of $\varphi$ is non-negative in complex directions tangent to $K^{\mathbb{C}}$-orbits in all points of $D$. Domains $D$ defined by orbit plurisubharmonic functions $\varphi$ are called orbit pseudoconvex.

Note that in this definition we do not require $\varphi$ to be plurisubharmonic (i.e. we do not suppose that the Levi form of $\varphi$ is non-negative in all complex directions).

Proposition (Heinzner-Sergeev [5]). Let $D$ be an orbit pseudoconvex domain in $\mathbb{C}^{n}$. Then it is orbit convex.

2. Matrix Reinhardt domains. As we have noted before, Reinhardt domains in $\left(\mathbb{C}^{*}\right)^{n}$ are orbit convex $\Leftrightarrow$ they are log-convex. Such domains in $\left(\mathbb{C}^{*}\right)^{n}$ are always orbit connected. We see that the orbit-convexity of Reinhardt domains is 
closely related to their holomorphic convexity — recall that a complete Reinhardt domain $D$ in $\mathbb{C}^{n}$ is holomorphically convex if and only if it is log-convex. Here $D$ is complete iff with any point $\left(z_{1}^{0}, \ldots, z_{n}^{0}\right)$ it contains also the polydisc $\left\{\left(z_{1}, \ldots, z_{n}\right)\right.$ : $\left.\left|z_{i}\right| \leq\left|z_{i}^{0}\right|, i=1, \ldots, n\right\}$. Any complete log-convex Reinhardt domain in $\mathbb{C}^{n}$ is orbit convex.

Let us consider now a generalization of Reinhardt domains to the matrix case. Denote by $\mathbb{C}^{n}[m \times m]$ the space of $n$ matrix variables, i.e. a point $Z \in \mathbb{C}^{n}[m \times m]$ is an $n$-tuple $Z=\left(Z_{1}, \ldots, Z_{n}\right)$ where all $Z_{i}, 1 \leq i \leq n$, are $m \times m$-matrices with complex entries.

Definition (Sergeev [12]). A domain $D \subset \mathbb{C}^{n}[m \times m]$ is called a matrix Reinhardt domain if with any point $\left(Z_{1}, \ldots, Z_{n}\right) \in D$ all points of the form

$$
\left(U_{1} Z_{1} V_{1}, \ldots, U_{n} Z_{n} V_{n}\right)
$$

for arbitrary unitary matrices $U_{i}, V_{i}, 1 \leq i \leq n$, also belong to $D$.

Otherwise, matrix Reinhardt domains are the domains invariant under the natural action of the group $[\mathrm{U}(m) \times \mathrm{U}(m)]^{n}$ on $\mathbb{C}^{n}[m \times m]$.

A matrix Reinhardt domain $D$ is, in general, not a Reinhardt domain in $\mathbb{C}^{n m^{2}}$ but we can always associate with $D$ a Reinhardt open set (maybe not connected) $\operatorname{diag} D$ in $\mathbb{C}^{n m}$, namely

$\operatorname{diag} D=\left\{\left(Z_{1}, \ldots, Z_{n}\right) \in D\right.$ :

$Z_{i}$ are complex diagonal $m \times m$-matrices, $\left.1 \leq i \leq n\right\}$

We have the following matrix analogue of the above assertions for Reinhardt domains.

Proposition. A matrix Reinhardt domain $D \subset[\mathrm{GL}(m, \mathbb{C})]^{n}$ is orbit connected. Hence, $D$ is orbit convex if it is a domain of holomorphy.

This proposition is a corollary of general results on invariant domains in homogeneous spaces collected in the following

THEOREM 4. (i) Let $H$ be a closed connected complex subgroup in $K^{\mathbb{C}}$ and $D$ is a $K$-invariant domain in the homogeneous space $K^{\mathbb{C}} / H$ provided with the left action of $K^{\mathbb{C}}$ on $K^{\mathbb{C}} / H$. Then $D$ is orbit connected.

(ii) Let $L$ be a closed connected subgroup in $K$ and $D$ is a $K$-invariant domain in the homogeneous space $K^{\mathbb{C}} / L^{\mathbb{C}}$. Then its holomorphic hull $E(D)$ is schlicht and orbit convex.

(iii) Suppose, in addition to the assumptions of (ii), that $(K, L)$ form a symmetric pair, i.e. there exists an involutive automorphism $\sigma$ of $K$ such that $K_{\sigma}^{0} \subset$ $L \subset K_{\sigma}$ where $K_{\sigma}$ is the subgroup of $K$ consisting of points fixed by $\sigma$ and $K_{\sigma}^{0}$ is its identity connected component. Then $D \subset K^{\mathbb{C}} / L^{\mathbb{C}}$ is holomorphically convex $\Leftrightarrow D$ is orbit convex.

The assertions of the theorem (and other results in this direction) are contained in the papers by Lasalle [8], Rothaus [11], Loeb [9], Cœuré-Loeb [2]. 
Passing to the case of general matrix Reinhardt domains $D$ in $\mathbb{C}^{n}[m \times m]$ we have the following analogue of the above assertion for Reinhardt domains: a complete matrix Reinhardt domain $D$ is holomorphically convex $\Leftrightarrow \operatorname{diag} D$ is holomorphically convex $\Leftrightarrow \operatorname{diag} D$ is log-convex (Sergeev [12]). Here, $D$ is complete iff with any point $\left(Z_{1}^{0}, \ldots, Z_{n}^{0}\right)$ it contains also the matrix polydisc $\left\{\left(Z_{1}, \ldots, Z_{n}\right)\right.$ : $\left.\left\|Z_{i}\right\| \leq\left\|Z_{i}^{0}\right\|, \quad i=1, \ldots, n\right\}$ where $\|Z\|=\max \left\{\right.$ eigenvalues of $\left.\sqrt{Z^{*} Z}\right\}$ is the spectral norm of a matrix $Z$. In fact, a stronger result is true.

TheOREM 5. Let $D$ be a matrix Reinhardt domain in $\mathbb{C}^{n}[m \times m]$. Then $D$ is holomorphically convex $\Leftrightarrow \operatorname{diag} D$ is a connected holomorphically convex Reinhardt domain in $\mathbb{C}^{m n}$.

This theorem is proved by Bedford-Dadok [1], the sufficient part is proved independently by Zhou [15]. (Another proof of this result was proposed in Fels [3].) Bedford-Dadok [1] also considered domains invariant under so called polar actions of classical groups and proved a similar criterion for their holomorphic convexity.

3. Extended matrix disc. Another important example of orbit convex domains is provided with the extended matrix disc - a domain which arises naturally in the quantum field theory.

The matrix disc is a domain $\Delta$ in the space $\mathbb{C}[2 \times 2]$ of the form

$$
\Delta=\{Z \in \mathbb{C}[2 \times 2]:\|Z\|<1\} .
$$

The condition $\|Z\|<1$ where $\|\cdot\|$ is the spectral norm of $Z$ (cf. above) is equivalent to the positive-definiteness of the Hermitian matrix $I-Z^{*} Z$. The matrix disc is invariant under the action of the group $K=\mathrm{SU}(2) \times \mathrm{SU}(2)$ given by

$$
Z \mapsto U Z V^{-1}, \quad Z \in \Delta, U, V \in \mathrm{SU}(2) .
$$

The action of the complexified group $K^{\mathbb{C}}=\operatorname{SL}(2, \mathbb{C}) \times \operatorname{SL}(2, \mathbb{C})$ on $\mathbb{C}[2 \times 2]$ is given by the same formula and the complexification $\Delta_{\mathbb{C}}$ is equal to

$$
\Delta_{\mathbb{C}}=K^{\mathbb{C}} \cdot \Delta=\{Z \in \mathbb{C}[2 \times 2]:|\operatorname{det} Z|<1\} .
$$

$\mathbb{C}[2 \times 2]$ (but not a Reinhardt domain in $\mathbb{C}^{4}$ ).

The extended matrix disc $\Delta_{n}^{\prime}$ is defined as

$\Delta_{n}^{\prime}=\left\{\left(A Z_{1} B^{-1}, \ldots, A Z_{n} B^{-1}\right) \in \mathbb{C}^{n}[2 \times 2]:\left(Z_{1}, \ldots, Z_{n}\right) \in \Delta^{n}, A, B \in \operatorname{SL}(2, \mathbb{C})\right\}$.

Otherwise speaking, we consider the matrix polydisc

$$
\Delta^{n}=\left\{\left(Z_{1}, \ldots, Z_{n}\right) \in \mathbb{C}^{n}[2 \times 2]:\left\|Z_{i}\right\|<1, i=1, \ldots, n\right\}
$$

with the diagonal action of the group $K=\mathrm{SU}(2) \times \mathrm{SU}(2)$ on $\mathbb{C}^{n}[2 \times 2]$

$$
\left(Z_{1}, \ldots, Z_{n}\right) \mapsto\left(U Z_{1} V^{-1}, \ldots, U Z_{n} V^{-1}\right), \quad U, V \in \mathrm{SU}(2) .
$$

Then $\Delta^{n}$ is invariant under $K$ and $\Delta_{n}^{\prime}$ coincides with the image of $\Delta^{n}$ under the diagonal action of $K^{\mathbb{C}}=\mathrm{SL}(2, \mathbb{C}) \times \mathrm{SL}(2, \mathbb{C})$ given by the above formula

$$
\Delta_{n}^{\prime}=\Delta_{\mathbb{C}}^{n}=K^{\mathbb{C}} \cdot \Delta^{n}
$$

Note that $\Delta_{n}^{\prime}$ is not a matrix Reinhardt domain (Zhou). 
There was a conjecture proposed by some mathematicians and physicists (I've heard about it from V. S. Vladimirov) asserting that the extended matrix disc is a domain of holomorphy. It is in fact a compact version of the well-known "extended future tube conjecture" from quantum field theory (cf. Sec. II.4 below). Using the results of Sec. I we can prove that the "extended matrix disc conjecture" is true.

Theorem 6 (Heinzner-Sergeev [5]). The extended matrix disc $\Delta_{n}^{\prime}$ is a domain of holomorphy in $\mathbb{C}^{n}[2 \times 2]=\mathbb{C}^{4 n}$ for any $n$.

The theorem follows from Theorems 1 and 2 from Sec. I if we prove that $\Delta^{n}$ is a $K$-invariant orbit convex domain of holomorphy in $\mathbb{C}^{4 n}$. To check its orbit convexity one could use the Proposition from Sec. II.1 above.

4. Extended future tube conjecture and non-compact groups. We formulate now the extended future tube conjecture (cf., e.g., Vladimirov [14]). To define the extended future tube $\tau_{n}^{\prime}$ we need to substitute the matrix polydisc $\Delta^{n}$ in the definition of $\Delta_{n}^{\prime}$ from the Sec. II.3 by the direct product of future tubes $\tau^{+} \times$ $\ldots \times \tau^{+}(n$ times $)=\tau_{n}^{+}$where $\tau^{+}=\left\{z=x+i y \in \mathbb{C}^{4}: y_{1}^{2}>y_{2}^{2}+y_{3}^{2}+y_{4}^{2}, y_{1}>0\right\}$. Also the $K^{\mathbb{C}}$-action on $\mathbb{C}^{n}[2 \times 2]$ should be substituted by the diagonal action of the identity component $L_{+}^{\mathbb{C}}$ of the complex Lorentz group $L^{\mathbb{C}}=\mathrm{O}(4, \mathbb{C})$. In other words, the extended future tube $\tau_{n}^{\prime}$ is defined by

$$
\tau_{n}^{\prime}=\left\{\left(\Lambda z^{(1)}, \ldots, \Lambda z^{(n)}\right) \in \mathbb{C}^{4 n}: z^{(i)} \in \tau^{+}, i=1, \ldots, n ; \Lambda \in L_{+}^{\mathbb{C}}\right\} .
$$

So in the case of the extended future tube $\tau_{n}^{\prime}$ the notations of Sec. I are interpreted as follows:

$$
\begin{aligned}
D & =\tau^{+} \times \ldots \times \tau^{+}(n \text { times })=\tau_{n}^{+}, \\
D_{\mathbb{C}} & =\tau_{n}^{\prime}=K^{\mathbb{C}} \cdot \tau_{n}^{+}, \\
K & =L_{+}^{\uparrow}, \text { identity component of } L=\mathrm{O}(1,3) \\
K^{\mathbb{C}} & =L_{+}^{\mathbb{C}}, \text { identity component of } \mathrm{O}(4, \mathbb{C}) .
\end{aligned}
$$

The extended future tube conjecture asserts that $\tau_{n}$ is a domain of holomorphy for any $n$. It is still open for $n>2$. We believe that the reason why it is still unproved is of quite general character related to the lack of a comprehensive geometric invariant theory for non-compact groups. In our situation it means that some of the results of Sec. I used in the proof of the compact version of the extended future tube conjecture do not extend to the non-compact group $K=L_{+}^{\uparrow}$. To be more precise, there exists an analogue of Theorem 1 from Sec. I for $\tau_{n}^{\prime}$ proved by Bargmann-Hall-Wightman (cf. Jost [7]). There is also an analogue of the Hilbert theorem used in the proof of Theorem 2, Sec. I - it is a theorem of Hall (cf. Jost [7]). However the Cartan theorem (also used in the proof of Theorem 2) is not true for non-compact groups as could be seen from the following simple example communicated to me by Zhou (cf. also Heinzner [4]). Take $D=\mathbb{C}^{2}$ with the action of $K=\mathbb{R}$ given by the representation

$$
\mathbb{R} \rightarrow \mathrm{GL}(2, \mathbb{C}), \quad t \mapsto\left(\begin{array}{ll}
1 & t \\
0 & 1
\end{array}\right)\left(\begin{array}{l}
z_{1} \\
z_{2}
\end{array}\right)=\left(z_{1}+t z_{2}, z_{2}\right) .
$$


Consider the analytic submanifold $A=\left\{\left(z_{1}, z_{2}\right) \in \mathbb{C}^{2}: z_{2}=0\right\}$ invariant under $\mathbb{R}$. Then $\mathbb{R}$-invariant analytic functions on $A$ are given by arbitrary holomorphic functions $f\left(z_{1}\right)$. However, $\mathbb{R}$-invariant holomorphic functions on $\mathbb{C}^{2}$ are the entire fucntions $F$ on $\mathbb{C}^{2}$ satisfying the condition

$$
F\left(z_{1}+t z_{2}, z_{2}\right)=F\left(z_{1}, z_{2}\right) \text { for any }\left(z_{1}, z_{2}\right) \in \mathbb{C}^{2}, t \in \mathbb{R} .
$$

In particular, such functions $F$ should be constant in $z_{1}$. Hence, the invariant version of Cartan's extension theorem is not true in this case.

\section{References}

[1] E. Bedford and J. Dadok, Generalized Reinhardt domains, J. Geom. Anal. 1 (1991), $1-17$.

[2] G. Cœuré and J. J. Loeb, Univalence de certaines envelopes d'holomorphie, C. R. Acad. Sci. Paris Sér. I 302 (1986), 59-61.

[3] G. Fels, Holomorphiehüllen der Reinhardtschen Gebiete sowie $U_{n}(\mathbb{C}) \times U_{n}(\mathbb{C})$ invarianten Matrizengebiete, Ruhr-Universität preprint, Bochum, 1990.

[4] P. Heinzner, Geometric invariant theory on Stein spaces, Math. Ann. 289 (1991), 631-662.

[5] P. Heinzner and A. G. Sergeev, The extended matrix disc is a domain of holomorphy, Math. USSR-Izv. 38 (1992), 637-645.

[6] G. Hochschild, The Structure of Lie Groups, Holden-Day, San Francisco, 1965.

[7] R. Jost, The General Theory of Quantized Fields, Amer. Math. Soc., Providence, R.I., 1965.

[8] M. Lasalle, Séries de Laurent des fonctions holomorphes dans la complexification d'un espace symétrique compact, Ann. Sci. Ecole Norm. Sup. (4) 11 (1978), 167-210.

[9] J. J. Loeb, Plurisousharmonicité et convexité sur les groupes réductifs complexes, Publ. IRMA Lille 2 (8) (1986).

[10] P. Pflug, Über polynomiale Funktionen auf Holomorphiegebieten, Math. Z. 139 (1974), 133-139.

[11] O. Rothaus, Envelopes of holomorphy of domains in complex Lie groups, in: Problems of Analysis, Princeton Univ. Press, Princeton, 1970, 309-317.

[12] A. G. Sergeev, On matrix Reinhardt and circled domains, in: Several Complex Variables. Proc. Mittag-Leffler Inst., Princeton Univ. Press, Princeton, 1993, 573-586.

[13] N. Sibony, Prolongement des fonctions holomorphes bornées et métrique de Carathéodory, Invent. Math. 29 (1975), 205-230.

[14] V. S. Vladimirov, Several complex variables in mathematical physics, in: Lecture Notes in Math. 919, Springer, Berlin, 1982, 358-386.

[15] X. Zhou, On matrix Reinhardt domains, Math. Ann. 287 (1990), 35-46.

[16] -, On orbit connectedness, orbit convexity, and envelopes of holomorphy, Math. USSRIzv., to appear. 